\title{
Analysis of a marine phototrophic biofilm by confocal laser scanning microscopy using the new image quantification software PHLIP Lukas N Mueller* ${ }^{*}, 2$, Jody FC de Brouwer ${ }^{\dagger 3}$, Jonas S Almeida ${ }^{2,4}$, Lucas J Stal $^{5}$ and João B Xavier 6
}

\begin{abstract}
Address: ${ }^{1}$ Institute for Molecular Systems Biology, ETH Hönggerberg, CH-8093 Zürich, Switzerland, ${ }^{2}$ Instituto de Tecnologia de Quimíca e Biológica, Universidade Nova de Lisboa, 2781-901 Oeiras, Portugal, ${ }^{3}$ Scottish Association for Marine Science, Oban, Argyll, Scotland, PA37 1QA, for Estuarine and Marine Ecology, Netherlands Institute of Ecology (NIOO-KNAW), PO Box 140, 4400 AC Yerseke, The Netherlands and ${ }^{6}$ Department of Biotechnology, Delft University of Technology, 2628 BC Delft, The Netherlands

Email: Lukas N Mueller* - lukas.mueller@imsb.biol.ethz.ch; Jody FC de Brouwer - jody.debrouwer@sams.ac.uk; Jonas S Almeida - almeidaj@musc.edu; Lucas J Stal - l.stal@nioo.knaw.nl; João B Xavier - j.xavier@tnw.tudelft.nl

* Corresponding author †Equal contributors
\end{abstract} UK, ${ }^{4}$ Department of Biostatistics, Bioinformatics and Epidemiology, Medical University of South Carolina, Charleston SC 29425, USA, ${ }^{5}$ Center

Published: 16 January 2006

BMC Ecology 2006, 6:1 doi:10.1 186/1472-6785-6-1

This article is available from: http://www.biomedcentral.com/l472-6785/6/I

(C) 2006 Mueller et al; licensee BioMed Central Ltd.

This is an Open Access article distributed under the terms of the Creative Commons Attribution License (http://creativecommons.org/licenses/by/2.0), which permits unrestricted use, distribution, and reproduction in any medium, provided the original work is properly cited.

\begin{abstract}
Background: Confocal laser scanning microscopy (CLSM) is the method of choice to study interfacial biofilms and acquires time-resolved three-dimensional data of the biofilm structure. CLSM can be used in a multi-channel modus where the different channels map individual biofilm components. This communication presents a novel image quantification tool, PHLIP, for the quantitative analysis of large amounts of multichannel CLSM data in an automated way. PHLIP can be freely downloaded from http://phlip.sourceforge.net.
\end{abstract}

Results: PHLIP is an open source public license Matlab toolbox that includes functions for CLSM imaging data handling and ten image analysis operations describing various aspects of biofilm morphology. The use of PHLIP is here demonstrated by a study of the development of a natural marine phototrophic biofilm. It is shown how the examination of the individual biofilm components using the multi-channel capability of PHLIP allowed the description of the dynamic spatial and temporal separation of diatoms, bacteria and organic and inorganic matter during the shift from a bacteria-dominated to a diatom-dominated phototrophic biofilm. Reflection images and weight measurements complementing the PHLIP analyses suggest that a large part of the biofilm mass consisted of inorganic mineral material.

Conclusion: The presented case study reveals new insight into the temporal development of a phototrophic biofilm where multi-channel imaging allowed to parallel monitor the dynamics of the individual biofilm components over time. This application of PHLIP presents the power of biofilm image analysis by multi-channel CLSM software and demonstrates the importance of PHLIP for the scientific community as a flexible and extendable image analysis platform for automated image processing. 


\section{Background}

Interfacial biofilms constitute an important reservoir of microbial life in aquatic systems. The main focus of biofilm research has been on the examination of bacterial biofilms (reviewed in [1]), which cause widespread problems in industrial fluid processing applications (reviewed in [2]) and play a major role in human infection diseases [3]. In general, growth of biofilms is initiated by the attachment of cells to a conditioned substratum, after which in situ growth of micro-organisms and production of extracellular matrix components build up the biofilm structure [4]. Many studies have investigated the physiology and structure of bacterial biofilms in order to understand the underlying processes of attachment, detachment and growth (reviewed in [5]).

In contrast to medical and industrial settings where biofilms generally develop in the dark, those present in freshwater and marine aquatic ecosystems are often exposed to sunlight. In these environments, biofilms are diverse species communities typically dominated by micro-algal consortia [6-9]. Phototrophic biofilms in aquatic environments represent an important carbon source for other trophic levels $[10,11]$ and affect mass transfer processes at the ecosystem scale [8].

Confocal laser scanning microscopy (CLSM) is an important method for the study of biofilm structure. Since its first application [12], CLSM has become widely used to improve the understanding of the biofilm architecture [13]. CLSM is a non-destructive and non-invasive method with the capability to provide time-resolved three-dimensional images of biofilms. In addition, multiple fluorescent channels can be recorded simultaneously, which offers the possibility to directly observe the development of individual biofilm components [14]. Multi-channel observations of phototrophic biofilms take advantage of autofluorescence of the micro-algae or record the fluorescence signal of biofilm components labeled with specific markers. Analysis of CLSM images has shown that biofilm communities form highly structured microbial assemblies $[15,16]$. Studies using CLSM have further confirmed that the development of biofilms depends on various factors including mass transport properties [17] and have shown the importance of metabolic interactions within the microbial communities themselves [18].

In many studies, the analysis of CLSM data has been of rather qualitative than quantitative nature and consisted entirely of a visual image inspection [19,20]. This approach is however subjective and not feasible when large quantities of data have to be analyzed, which is often necessary to ensure the significance of the outcome of the analyses [21]. For quantitative analysis of CLSM data, computer software with different functionalities ranging from cell number counting [22] to the classification of bacteria morphotypes [23] are currently available and are increasingly being used. In order to address the necessity of a more directed morphological quantification of biofilm data, image analysis programs such as COMSTAT [24] and ISA [25] were developed to quantitatively analyze single channel 3D CLSM data of biofilm imaging by determining a set of morphological parameters. Despite the wide use of the available image analysis software in morphological investigations of bacterial biofilms $[15,21,26,27]$, their application is restricted to the analysis of single-channel CLSM data at a time. This singlechannel limitation falls short of the capabilities of modern CLSM devices and is a significant disadvantage since multi-channel images have to be quantified in separate analysis sessions and multiple channel analysis distinguishing various biofilm components cannot be approached comprehensively.

This communication describes a new image quantification package, PHLIP (PHobia Laser scanning microscopy Imaging Processor), which enables analysis of multichannel CLSM data. PHLIP was developed in the scope of the EU/FP5 funded project PHOBIA (Phototrophic Biofilms and Its potential Applications [28]) and extends previous work on automated CLSM image analysis [29,30] by including a new set of tools to automatically quantify CLSM imaging in biofilm systems, necessary to produce statistically meaningful results. PHLIP is available as an open source project (see Materials and Methods) and encourages users to extend the program's capabilities. The potential of PHLIP is illustrated here in a study of the temporal development of a natural phototrophic biofilm.

\section{Implementation}

The image analysis software PHLIP was implemented as a MATLAB package running under MATLAB (Release 13, The Mathworks ${ }^{\mathrm{TM}}$ ) on Windows, Linux and OSX platform and does not require additional toolboxes. PHLIP is released under an open source license and current software versions as well as the documentation and example image datasets can be downloaded from the project webpage [31]. The architecture of the program was developed with flexibility and extensibility in mind and its functionality can be easily expanded with new features. PHLIP therefore represents a platform for the integration of novel image processing operations without the need to code for import, export or preprocessing functions. Supplementary information regarding the program structure and guidance for the implementation of new image operations are available on the project homepage. Fig. 6 describes the data analysis flow of PHLIP and illustrates the individual image processing functions of the 5 distinct modules constituting the program. 
Table I: Minimum and maximum values for the image analysis parameters calculated by PHLIP during the development of phototrophic marine biofilms. ANOVA testing determined whether variations in biofilm parameters over time was statistically significant. Significance levels are indicated by asterisks. Channels; concanavaline A (ConA), syto64 (syto), chlorophylla (chla).

\begin{tabular}{|c|c|c|c|c|c|c|c|c|c|c|c|c|c|c|}
\hline & \multicolumn{6}{|c|}{ Single channel } & \multicolumn{6}{|c|}{ Two channels } & \multicolumn{2}{|l|}{ All channels } \\
\hline & ConA & & syto & & Chla & & ConA-syto & & ConA-c & chla & syto-chla & & & \\
\hline Biovolume $^{a}$ & $5.8-28.8$ & n.s. & $0.5-4.7$ & $* * *$ & $0.1-61.9$ & $* * *$ & & & & & & & $8.4-82.3$ & $* * * *$ \\
\hline Substratum coverage & $0.48-0.86$ & n.s. & $0.07-1.63$ & $* * *$ & $0.03-2.86$ & $* * *$ & & & & & & & & \\
\hline Area-to-volume ratio & $3.5-5.8$ & n.s. & $3.2-5.0$ & ** & $1.4-4.4$ & $* *$ & & & & & & & & \\
\hline Horizontal spreading ${ }^{a}$ & $8.4-9.1$ & n.s. & $5.8-8.3$ & $* *$ & 1.9-7.7 & $* * *$ & & & & & & & & \\
\hline Vertical spreadinga & $0.09-27.5$ & $* * *$ & $0.03-24.0$ & $* *$ & $0.02-30.4$ & $* * *$ & & & & & & & & \\
\hline Total spreading ${ }^{a}$ & $8.5-11.7$ & n.s. & $8.3-9.3$ & * & $2.0-10.8$ & $* * *$ & & & & & & & & \\
\hline Average thickness & $15.3-127.4$ & $* * *$ & $6.3-111.2$ & $*$ & $4.3-102.3$ & $* * *$ & & & & & & & $6.3-111.2$ & $* *$ \\
\hline Biofilm roughness & $0.15-0.26$ & n.s. & $0.04-0.20$ & $* * *$ & $0.02-0.30$ & $* * *$ & & & & & & & $0.24-0.35$ & n.s. \\
\hline Co-localization in $2 \mathrm{D}$ & & & & & & & $5.4-14.5$ & n.s. & $0-3.7$ & n.s. & $0.5-27.9$ & n.s. & & \\
\hline Co-localization in 3D & & & & & & & $0.9-5.2$ & n.s. & $0-0.8$ & n.s. & $0.04-2.7$ & n.s. & & \\
\hline
\end{tabular}

$a: \times 10^{3} ; * * * ;<<0.001 ; * *: p<0.01 ; *: p<0.05 ;$ n.s. not significant

\section{PHLIP-ML, a new standard for CLSM imaging data}

Due to the lack of uniformity in image formats between the different CLSM vendors or even between different models from the same vendor, a new CLSM standard formatted as XML (eXtensible Markup Language) was developed. XML was chosen as its general acceptance as a standard to describe data in a vast number of fields [32], including generous support for development of converter from other formats. Basic image acquisition parameters (image resolution, scanning step etc.) and CLSM imaging data information (file names, number of channels etc.) are stored in the PHLIP-ML files. Optionally, PHLIP image analysis results can also be incorporated into PHLIP-ML. Detailed information about the PHLIP-ML data structure as well as its Document Type Definition file (dtd), graphical representations and example data sets can be retrieved from the PHLIP-ML webpage [33]. A universal converter data2xml is available on the PHLIP project homepage [34] and offers automated conversion of PHLIP supported CLSM formats into PHLIP-ML. For not supported CLSM formats, data2xml can be used to generate in an interactive process PHLIP-ML files from any kind of microscopic images without any previous XML knowledge.

\section{Import and pre-processing of CLSM data}

In addition to the PHLIP-ML format, PHLIP currently offers direct support for the two CLSM models of Leica ${ }^{\mathrm{TM}}$ (Heidelberg, Germany), Leica TCS-NT and Leica TCS4D. The latter format is also used by the COMSTAT program [24], rendering PHLIP compatible to COMSTAT. In addition, the functionality of PHLIP can be easily extended to allow direct import of data from other CLSM formats. In the present version, PHLIP enables quantification of CLSM data sets containing up to four fluorescent channels.
The image pre-processing module offers several possibilities to pre-process the batch imaging data. A batch editing menu allows the user to select or remove image stacks from the data set. To save computer memory and reduce computational requirements, the cross section resolution of CLSM image stacks can be adjusted by skipping image cross sections within image stacks (Fig 6A). Furthermore, the microscope scanning direction can be defined which is important for the correct $3 \mathrm{D}$ reconstruction of image stacks from image cross sections files (Fig 6B). Additional pre-processing functions include adjustment of the biofilm carrier horizontal position within the 3D image stack (Fig 6C), which allows to define the deepest cross section containing any biofilm material, and pre-reading of the input files to ensure data availability.

\section{Thresholding and graphical user interface}

Image segmentation is the process where every pixel with a grey value above a given threshold is classified as foreground and a pixel with value below a given threshold as background, resulting in a binary image. Selection of a threshold level is therefore an important step in the quantitative analysis of CLSM biofilm imaging as altering the threshold value will change the volume and morphology assigned to a given biofilm component [29]. Threshold determination is typically carried out by either manual assessment (e.g. $[25,35,36]$ ) or automated methods $[29,37,38]$. To our knowledge, there is no generally accepted method, manual or automated, that works accurately for every possible set of imaging data. For a discussion on the applicability of automatic thresholding see a study by Yang et al [37] in which several automated threshold selection methods are evaluated.

Automated thresholding algorithms have several advantages over manual selection methods but in our view there is no automated procedure that guaranties to work cor- 
Table 2: Summary of the excitation wavelengths and emission filters for the multichannel signal of the CLSM using band pass (BP) and long pass (LP) filter.

\begin{tabular}{|c|c|c|}
\hline Staining & $\begin{array}{c}\text { Excitation } \\
{[\mathrm{nm}]}\end{array}$ & $\begin{array}{c}\text { Emission filter } \\
{[\mathrm{nm}]}\end{array}$ \\
\hline concanavaline A & 488 & $580 \pm 30(\mathrm{BP})$ \\
\hline syto64 & 568 & 600 (BP) \\
\hline $\begin{array}{l}\text { chlorophyll } a \\
\text { (autofluorescence) }\end{array}$ & 647 & 665 (LP) \\
\hline
\end{tabular}

rectly with every image set. This is partially because of the wide variability in characteristics of images from different samples, e.g. in terms of image histograms or spatial distribution of features within the samples. Advantages of automated thresholding methods include the possibility of automating the full image processing procedure [30]. PHILP has been equipped with this automating capability allowing the processing of large image datasets without user intervention and eliminating subjectivity associated with visual threshold selection by an operator $[29,38]$. However, the use of automated threshold methods should always be complemented with some degree of supervision, which reinforces the critical value of multichannel visualization methods to inspect the computed thresholds.

PHLIP offers users the choice of both manual and automated threshold selection. For manual threshold selection, PHLIP provides a multi functional graphical user interface (GUI) to assist in the visual determination of the threshold level to be used for every color channel of each dataset. For automated threshold selection, PHLIP implements a 3D extension of the Otsu algorithm [29]. Otsu is a well-established method that consists of a simple threshold selection procedure and, therefore, does not constitute a significant computationally burden to the image processing as a whole. This renders the method particularly suitable to image analysis systems, which will most likely be installed on personal computers. The method is based on discriminate analysis, which determines the variable that separates best between two naturally occurring groups. In the application of image analysis, a grey level value is selected, which separates the two groups of foreground and background grey level values. In PHLIP, the 3D Otsu threshold selection is applied to the CLSM data set to calculate thresholds independently for each color channel in each image stack.

With the threshold level being selected either manually or automatically, the GUI allows the user to view the different channels of an image stack and to browse through their cross sections, assessing the adequacy of the determined thresholds. Three different views of the image stack cross sections help to set, alter or check a threshold; an original grey level view, a view of the thresholded image and a combined view where the thresholded image overlays the original grey level image.

\section{Morphological characterization}

Following binarization of the image through thresholding, biofilm parameters are calculated from the binary image stacks with a prior optional connected volume filtration (CVF) $[24,30]$. The CVF method removes "floating" foreground pixels, ensuring that every foreground pixel is connected to the substratum through the connection to other neighboring pixels. Application of the CVF operation is optional, as users may prefer to include relevant floating material in their quantitative analysis depending on the characteristics of the system being analyzed. Presently, PHLIP can quantify 10 different biofilm features, which are classified into 6 single-channel $(A-F)$, 2 two-channel (G, H) and 5 all-channel measurements.

A. Biovolume: the biovolume, $\mathrm{V}$, is the number of foreground pixels, $\mathrm{N}$, in an image stack multiplied by the voxel volume, which is defined as the product of the squared pixel size, $\mathrm{px}$, and the scanning step size, zStep [35].

B. Substratum coverage: PHLIP calculates the fraction of pixels occupied by biofilm material for each image cross section [30]. The fraction, $F(z)$, is defined as the ratio of foreground pixels to the total number of pixels for a given cross section and is then transformed to percentage, $\mathrm{C}(\mathrm{z})$.

C. Area to volume ratio: the surface area, A, of an image stack is the number of foreground pixels which are connected to at least one neighboring background pixel. The final value is then obtained by calculating the ratio $\mathrm{A}$ to $\mathrm{V}$ [24].

D. Spatial spreading: this function characterizes the spreading of biovolume in space. Three different values are calculated; horizontal spreading (in $\mathrm{xy}$ direction), $\mathrm{S}_{\mathrm{xy}^{\prime}}$ vertical spreading (in $z$ direction), $S_{z^{\prime}}$ and total spreading (in xyz space), $\mathrm{S}_{\mathrm{xyz}}$. Initially, three distributions $\mathrm{D}_{\mathrm{x}^{\prime}} \mathrm{D}_{\mathrm{y}}$ and $\mathrm{D}_{\mathrm{z}}$ containing the values of the $\mathrm{x}, \mathrm{y}$ or $\mathrm{z}$ coordinates of each foreground pixel in a given image stack are created. The variances $\sigma_{\mathrm{x}^{\prime}} \sigma_{\mathrm{y}}$ and $\sigma_{\mathrm{z}}$ are then calculated from $\mathrm{D}_{\mathrm{x}^{\prime}} \mathrm{D}_{\mathrm{y}}$ and $D_{z} \cdot S_{z}$ results directly from $\sigma_{z}(1)$ while equation 2 combines $\sigma_{\mathrm{x}}$ and $\sigma_{\mathrm{y}}$ to obtain $\mathrm{S}_{\mathrm{xy}}$. $\mathrm{S}_{\mathrm{xyz}}$ is determined by $\sigma_{\mathrm{x}^{\prime}}$ $\sigma_{\mathrm{y}}$ and $\sigma_{\mathrm{z}}(3)$. All three parameters are dimensionless:

$\mathrm{S}_{\mathrm{z}}=\sigma_{\mathrm{z}}$

$S_{x y}=\sigma_{x}^{2}+\sigma_{y}^{2}$

$S_{x y z}=\sigma_{x}^{2}+\sigma_{y}^{2}+\sigma_{z}^{2}$ 
E. Mean thickness and roughness: these parameters are widely used to describe the morphology of the biofilms. The function first applies a height projection transformation to the image stack where for every point in the $x y$ plane the maximal height $\mathrm{h}$ of the corresponding foreground pixels in $\mathrm{z}$ direction is stored. The average of the resulting distribution of pixel height $h$ is then calculated and represents the mean thickness, $M,[30]$. To obtain the roughness coefficient, $\mathrm{R}$, the standard deviation of the distribution is divided by $M$ [24].

F. Fractal dimension in 2D: the fractal dimension parameter calculates a value that varies between 1 and 2 and describes the roughness of the biofilm boundary between foreground and background pixel in a cross-section at height $\mathrm{z}$. Higher values of the fractal dimension parameter indicate a rougher biofilm boundary. The fractal dimension parameter is calculated as described by Yang et al [25].

G. Co-localization in 3D: the co-localization in 3D operation calculates the percentage of overlapping biovolume in two selected channels. The co-localization volume, $\mathrm{V}_{\text {col }}$ is calculated by counting the presence of foreground pixels located at identical xyz positions in two channels. This is divided by the total biovolume of both channels [39].

H. Co-localization in 2D: the co-localization can also be studied in 2D for each cross section located at height $\mathrm{z}$. Analogous to operation $\mathrm{F}$, the fraction of co-localized to the total occupied area in 2D of two channels is calculated for every cross section [39].

All channels operations: biovolume, substratum coverage, area to volume ratio, spatial spreading, mean thickness and roughness analyses in the all channel mode are calculated by the same mathematics as their single channel operation analogues A to E. These operations work with a new 3D stack containing all biofilm elements from each channel where overlapping elements between the different channels are removed to avoid double counting.

\section{Data output}

PHLIP offers the possibility to save the calculated results in HTML, text and PHLIP-ML format. The HTML output is a formatted file presenting the results in tables and also contains plots for operations $\mathrm{B}, \mathrm{F}$ and $\mathrm{H}$. This data file can be opened by most spreadsheet programs and facilitates further analysis of the obtained results. Alternatively to the HTML format, analysis results can be formatted in a tab-delimitated matrix into a simple text file. As described above, the calculated results can also be saved in XML format using the PHLIP-ML data structure. This feature has the advantage that defined thresholds from an earlier analysis are stored in the PHLIP-ML file and can be re- loaded into PHLIP to continue a previous analysis procedure. In addition, PHLIP-ML formatted CLSM analysis results represent a compact way to archive imaging data or to transfer calculated results to other downstream data analysis applications.

\section{Experimental set-up for the growth of phototropic biofilms} Natural phototrophic biofilms were grown in 701 containers that were fed by a flow of fresh unfiltered natural seawater (Oosterschelde, the Netherlands) at a dilution rate of $0.43 \mathrm{~h}^{-1}$. Microscope glass slides were cleaned using $70 \%$ ethanol, dried and subsequently placed vertically in the water. The system was exposed to a 14:10 h light:dark regime at an incident photon irradiance of $40 \mu \mathrm{mol} \cdot \mathrm{m}^{-}$ $2 \cdot \mathrm{s}^{-1}$. Pumps circulating water at a rate of $3001 \cdot \mathrm{h}^{-1}$ ensured continuous turbulent mixing. Sampling was performed over a period of 44 days. On 7 sampling days $(\mathrm{T}=$ $3,5,10,18,25,31,44$ days), 3 glass slides were randomly removed from the water reservoir $4 \mathrm{~h}$ after the onset of light and prepared for CLSM analysis.

Glass slides were rinsed twice with $1 \mathrm{ml}$ of PBS (phosphate buffered saline: $0.4 \mathrm{~g} \cdot \mathrm{l}^{-1} \mathrm{KC} 1,12.6 \mathrm{~g} \cdot \mathrm{l}^{-1} \mathrm{NaC} 1,1.6$ $\mathrm{g} \cdot \mathrm{l}^{-1} \mathrm{Na}_{2} \mathrm{HPO}_{4} \cdot 2 \mathrm{H}_{2} \mathrm{O}, 0.25 \mathrm{~g} \cdot \mathrm{l}^{-1} \mathrm{KH}_{2} \mathrm{PO}_{4}, \mathrm{pH}=8$ ). Excess fluid was removed by using Whatman $\mathrm{GF} / \mathrm{B}$ filter paper (Maidstone, UK). Subsequently, the slides were incubated in the dark for 20 min using $200 \mu$ lof the lectin concanavaline A (ConA, Molecular Probes, Eugene, Oregon, USA) at a concentration of $100 \mu \mathrm{g} \cdot \mathrm{ml}^{-1}$ (in PBS). Excess ConA was removed by two rinses of $1 \mathrm{ml}$ PBS and one rinse with $1 \mathrm{ml}$ TRIS (18.6 $\mathrm{g} \cdot \mathrm{l}^{-1}$ EDTA, $0.158 \mathrm{~g} \cdot \mathrm{l}^{-1}$ TRIS, $20 \mathrm{~g} \cdot \mathrm{l}^{-1}$ $\left.\mathrm{NaC} 1,0.4 \mathrm{~g} \cdot \mathrm{l}^{-1} \mathrm{KC} 1\right)$. Thereafter, slides were incubated for 2 min using $200 \mu \mathrm{l}, 5 \mathrm{mM}$ syto64 (Molecular Probes) dissolved in TRIS-buffer to stain bacteria. Excess syto64 was removed by 2 rinses of $1 \mathrm{ml}$ TRS and one rinse of $1 \mathrm{ml}$ PBS.

CLSM was performed using a TCS-NT microscope (Leica, Heidelberg, Germany) equipped with an Argon-Krypton laser. Biofilms were examined using a HCX APO L $40 \times 0.8$ water-immersion lens. Table 2 summarizes excitation wavelengths and emission filters of the CLSM. Seven random spots were measured at each of the 3 replicate glass slide, resulting in 21 replicate measurements per sampling day. In addition, reflection images were measured on selected biofilm samples. For this purpose, an RT 30/70 filter was used and the image stacks were recorded under identical conditions (i.e. similar area and vertical resolution) compared to the 3 fluorescence channel analysis.

\section{Statistical analyses}

One and two way nested analysis of variance (ANOVA) designs were used to test the effects of days and sampling slides on the biofilm parameters obtained from the PHLIP analysis. For one way ANOVA, slides were nested within 

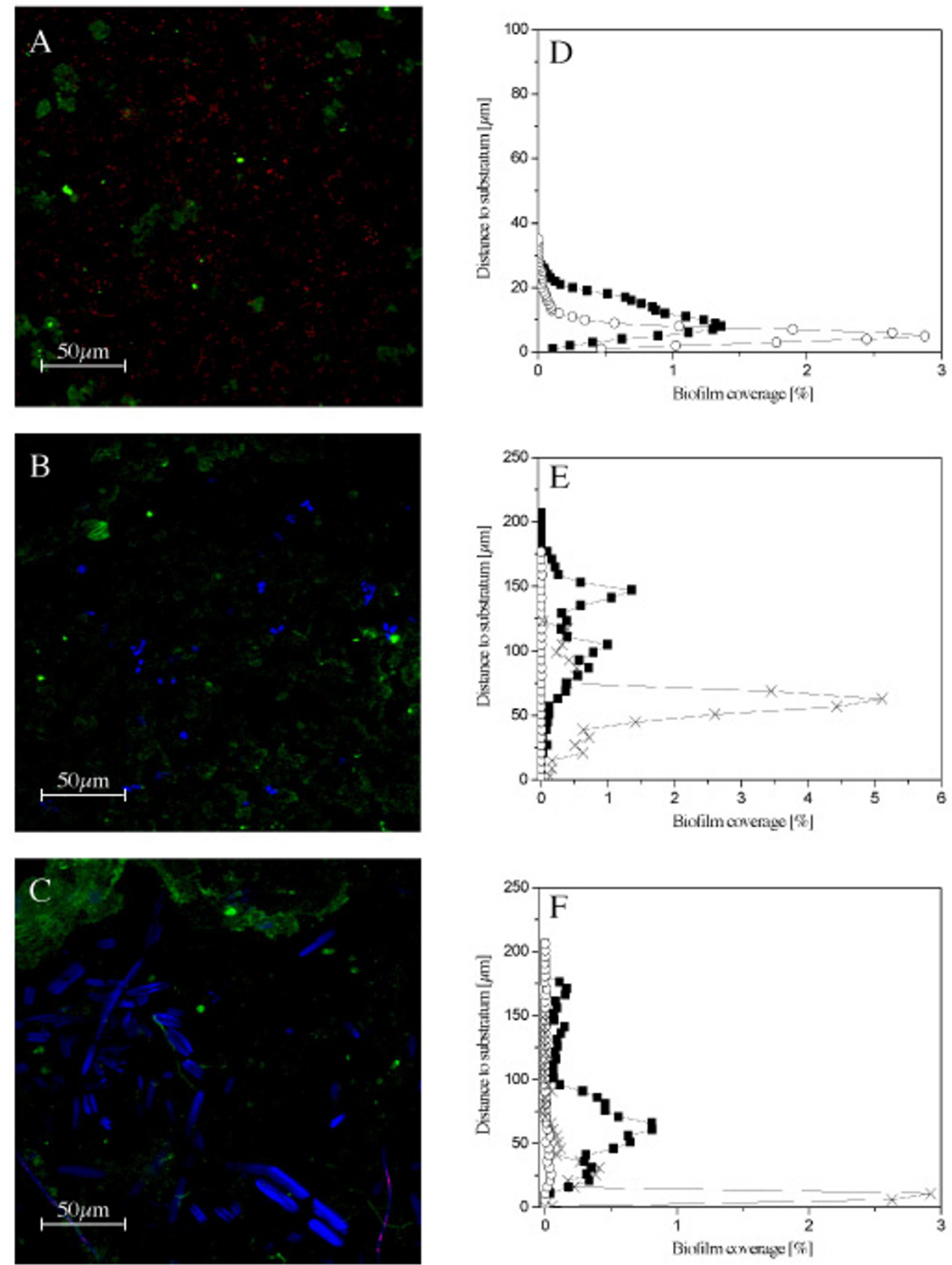

\section{Figure I}

Maximum intensity images $(A, B, C)$ and vertical profiles of biofilm coverage analyzed by PHLIP (D, E, F) of a biofilm grown on glass slides in natural seawater (Oosterschelde, the Netherlands). Images and vertical profiles are shown for representative samples measured at days $3(A, D)$, day $25(B, E)$ and day $44(C, F)$. The red channel and open circles represent bacteria stained with syto64, the green channel and black squares represent EPS labeled with ConA and the blue channel and crosses show chlorophyll $a$ autofluorescence. CLSM maximum intensity images represent areas of $250 \cdot \mathrm{m} \times 250 \cdot \mathrm{m}$. 

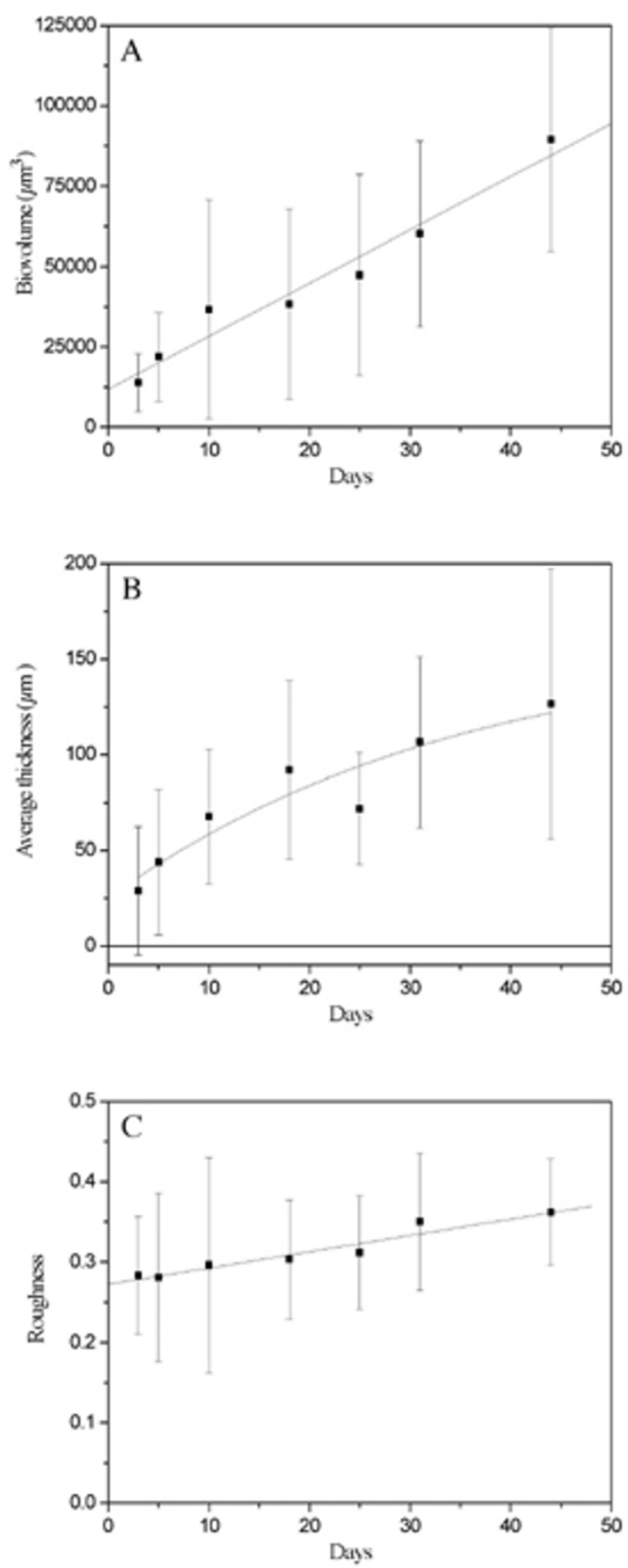

\section{Figure 2}

Temporal development of a natural phototrophic biofilm grown on glass slides in natural seawater (Oosterschelde, the Netherlands). All-channel image analyses of (A) biovolume, $(B)$ average thickness and $(C)$ roughness. Values indicate mean \pm S.D., $(n=2 I)$. days. For two way ANOVA, slides were nested within the interaction between days and channels. Analyses were performed on box-cox transformed data [40]. In addition, regression analysis was performed on the all-channel parameters. Either linear or exponential decay functions were used to fit the datasets. Analyses of variance were performed using Statistica 6.1, while regression analysis was performed using the software package Origin 6.0.

\section{Results}

The functionality of PHLIP is illustrated here by analyzing stacks of images acquired by CSLM at different maturation stages of a phototrophic biofilm. The quantitative description of biofilm morphogenesis with regard to the distribution of three dominant components, bacteria, micro-algae and extracellular polymeric substances (EPS), was pursued with the tandem goal of describing the biological process and establishing a multi-channel image analysis methodology.

A selection of maximum intensity projection images and the accompanying vertical profiles from image stacks recorded at different time points during the experiment are shown in Fig. 1. After a period of 3 days (Fig. 1A), biofilms were dominated by bacteria that were partly embedded in ConA-labeled EPS of the biofilm matrix. During the course of the experiment the contribution of bacteria decreased while the biomass of micro-algae increased. Changes in the morphology of the chloroplasts indicated a succession in the algal community with a general shift towards larger species (Fig. 1B,C). The presence of EPS was observed throughout the time series. EPS structures were visible mainly as bright spots or amorphous organic material. Occasionally, cell surface material of algal cell was stained with ConA. The thickness of the biofilms increased from about $30 \cdot \mathrm{m}$ after 3 days of incubation (Fig. 1D) to $200 \cdot \mathrm{m}$ in 44 -day-old biofilms (Fig. 1F). Bacteria were only observed as a significant fraction in the 3day-old biofilm where they were present near the substratum (Fig. 1D). The vertical distribution of micro-algae was variable. Part of the algal community was present close to the substratum while the remaining part extended into the biofilm (Fig. 1E,F). Vertical profiles showed considerable variation between different measurements, but EPS generally formed the thickest and outermost layer of the biofilm. A maximum of approximately $5 \%$ of the available surface was occupied by biofilm components after 44 days of incubation.

In a more comprehensive approach, the development of the biofilm was analyzed by CLSM measuring 7 replicate points on each of 3 replicate glass slides. Image analysis results (Table 1) were in good agreement with qualitative observations. The single channel parameters showed the temporal development in chlorophyll $a$ and syto64 signal 

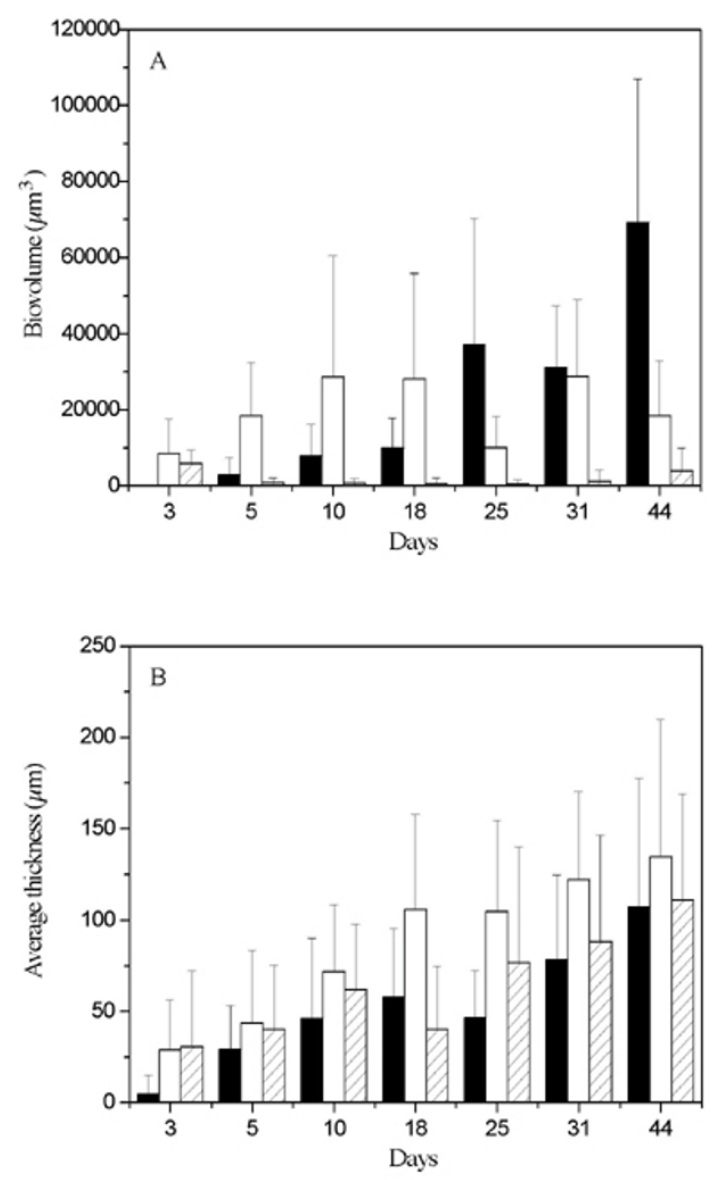

Figure 3

Temporal development of a natural phototrophic biofilms grown on glass slides in natural seawater (Oosterschelde, the Netherlands). Image analyses of separate channels representing chlorophyll $a$ fluorescence (black bars), EPS stained with ConA (open bars) and bacteria stained with syto64 (dashed bars). (A) biovolume and (B) average thickness. Values indicate mean \pm S.D., $(n=21)$.

representing growth of the phototrophic community and the decrease of bacteria, respectively. Growth of the micro-algae dominated the development of biofilm. This led to a significant increase in biovolume, thickness and roughness of the biofilm (Fig. 2A,B,C). Biomass-related and morphological parameters of ConA-stained organic matter did not vary over time while those related to the vertical distribution (i.e. average thickness and vertical spreading) increased significantly. Two channel operations indicated that co-localization between channels was generally low with up to $5.2 \%$ co-localization occurring in the three dimensional image stacks. A general trend in the results of the co-localization analysis showed that colocalization of channels ConA and syto64 was always higher then in channels ConA and chlorophyll $a$. This identifies a preferential spatial association of EPS with bacteria in relation to association of EPS with diatom, suggesting a bacterial origin of EPS.

The quantitative description of biofilm morphology performed by PHLIP analysis enables the testing for changes of distinct quantifiers in response to different condition. Equally, PHLIP allows to characterize the variability of these quantifiers within or between distinct biofilm communities. Statistical evaluation of the dataset by nested ANOVA, which was used here to illustrate the functionality of PHLIP, revealed significant differences between the slides as well as an effect of time. The results of these experiments will demonstrate the insights of the quantitative parameters extracted from CLSM image analysis. Temporal variability was observed for the all-channel operations as well as for the separate CLSM channels. This suggested that the placement of the slides in the experimental setup was a factor that determined the development of the biofilms, probably cause by spatial variations in light and/or flow conditions. However, by using the nested ANOVA-design it was possible to discriminate between different sources of variation. Hence, statistically meaningful results were obtained with respect to the variables time and channels. All-channel analyses resulted in a significant linear increase in biovolume (Fig. $2 \mathrm{~A}, \mathrm{R}^{2}=$ 0.9704, p < 0.001). Biofilm thickness (Fig. 2B) also increased over time $(\mathrm{p}<0.01)$ but the temporal evolution was best predicted by an exponential decay function $\left(\mathrm{R}^{2}=\right.$ $\left.0.8855, \mathrm{y}=0.33-0.29 \times \mathrm{e}^{(-\mathrm{x} / 16647)}\right)$. Even though a linear correlation between roughness and days of incubation (Fig. $2 \mathrm{C}, \mathrm{R}^{2}=0.9328$ ) was observed, statistical analysis showed that this trend was not significant $(\mathrm{p}=0.57)$.

Analysis of the information from different channels acquired by CLSM revealed that different biological components in the biofilms (i.e. bacteria, micro-algae and the ConA-stained EPS) followed different dynamics in time (Fig. 3, 4). Biovolume of the EPS was approximately constant over time $(\mathrm{p}=0.45)$, while the bacterial signal decreased ( $\mathrm{p}<0.001)$. In contrast, a steady increase $(\mathrm{p}<$ 0.001 ) was observed for the chlorophyll $a$ biovolume (Fig. 3A). Average thickness of the biofilm, as determined from each individual component, increased over time for all channels ( $<<0.001$ for chlorophyll $a$ and ConA, p < 0.05 for syto64), but thickness of ConA was significantly higher compared to the chlorophyll $a$ and syto64 signals ( $p<0.001$ and $\mathrm{p}<0.05$, Fig. 3B). This indicated that some EPS always exists in the region of the biofilm above the other components monitored, i.e. micro-algae and bacteria.

Variations in the morphological parameters area-to-volume ratio and biofilm roughness occurred over time (Fig. 

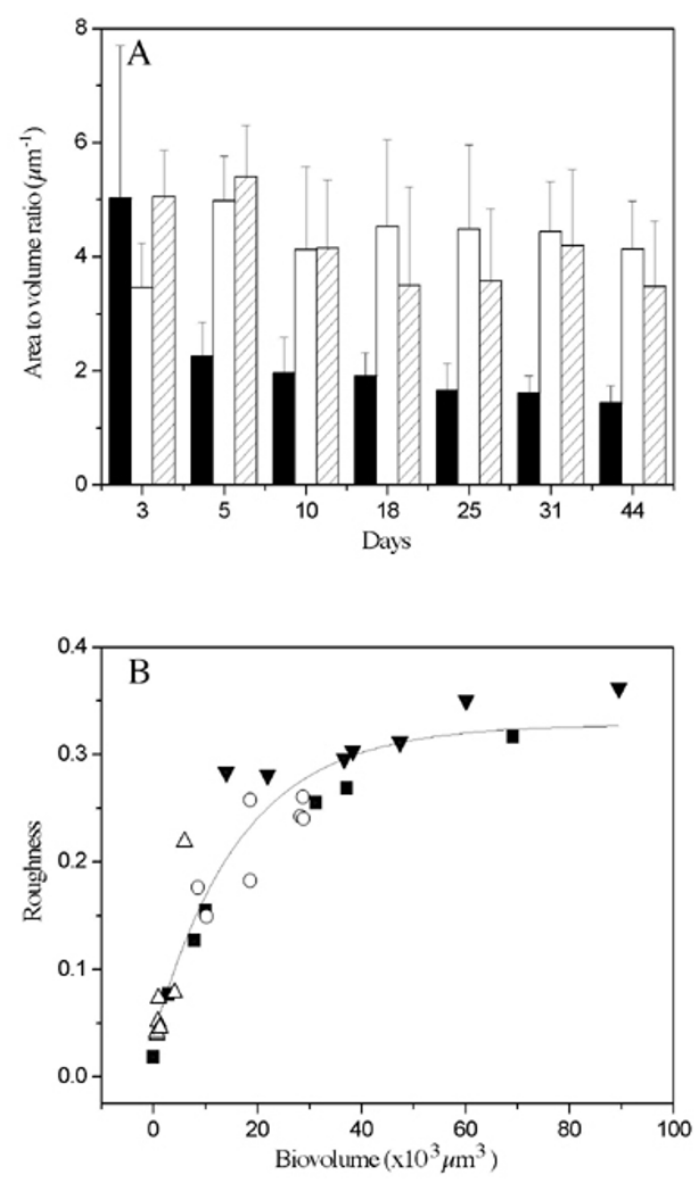

\section{Figure 4}

Development of morphological parameters analyzed from a phototrophic biofilm grown on glass in natural seawater (Oosterschelde, the Netherlands). (A) Temporal development of area-to-volume ratio. Separate channels represent chlorophyll a fluorescence (black bars), EPS stained with Con A (open bars) and bacteria stained with syto64 (dashed bars). Values indicate mean $\pm S . D,(n=2 I)$. (B) The correlation between biofilm roughness and biovolume. Filled squares: chlorophyll a fluorescence, open circles: ConA-fluorescence, open triangles: syto64, filled triangles, all channel signal.

4). Area-to-volume ratios of chlorophyll $a$ showed a steady decrease after 5 days of incubation $(\mathrm{p}<0.001$, Fig. $4 \mathrm{~A}$ ). This is in agreement with the changes in morphology of the chloroplasts as observed in the CLSM images (Fig. 1 ), indicating a succession of diatoms over time from small species to larger species. In addition, area-to-volume ratios of the bacterial signal varied over time $(\mathrm{p}<0.05)$ although no clear temporal trend was found. Finally, the EPS signal was constant and showed a rather diffuse distribution of signal compared to the chlorophyll $a$ autofluorescence. Biofilm roughness of the separate biofilm components gave comparable patterns to the temporal changes in biovolume. When the estimates of biofilm roughness were plotted as a function of biovolume, a correlation was found between the parameters (Fig. 4B). The exponential decay function that was fitted through the data explained $91 \%$ of the variation.

In addition to the quantification of biological components, which included bacteria, micro-algae and EPS, reflection images of biofilms visualize solid inorganic material (Fig. 5) and can be similarly analyzed using PHLIP as illustrated here. The quantified signal reflects the surface of the three dimensional objects from the inorganic material. Different reflective materials were distinguished including the bare glass substratum (I), silica frustules of diatoms present in the biofilm (II) and considerable amounts of amorphous material (III). A comparison of vertical profiles of the organic and inorganic parts of the biofilm indicated that the reflection signal (indicative for inorganic material) was consistently higher than the organic matter throughout the depth of biofilm. Biovolume estimates showed that for the image stack in Fig. 5, about $85 \%$ of the biofilm constituted of inorganic reflective material, while organic material represented the remaining $15 \%$ of the total biovolume. This percentage should be regarded as a rough estimate because inorganic particles represent opaque surfaces that are not penetrated by the laser. In addition, the glass surface gives a strong reflectance signal that is not a part of the biofilm itself. The value for the inorganic matter contribution of $85 \%$ agrees with determinations of dry weight and ash free dry weight contents of the biofilm (data not shown). These measurements indicated that the contribution of inorganic material to the biofilm was $58 \%$ after 3 day of incubation and increased to an average value of $78 \pm 4 \%$ during the course of the experiment. Hence, both confocal as well as dry mass determinations indicate a significant contribution of inorganic material in this phototrophic biofilm.

\section{Discussion}

This section separately covers the results describing the biofilm development followed by a discussion of the quantitative methodology implemented in PHLIP.

\section{Biofilm development}

The temporal development of a natural phototrophic biofilm was characterized by a linear increase in biovolume. Biofilm thickness increase leveled slightly off with time, which is explained by the fact that the laser of the CLSM did not penetrate anymore all the way down to the substratum of the biofilm. Eventually, phototrophic microorganisms (dominated by diatoms) became the dominant component of this biofilm. A succession of the phototrophic community was evident from small to larger 

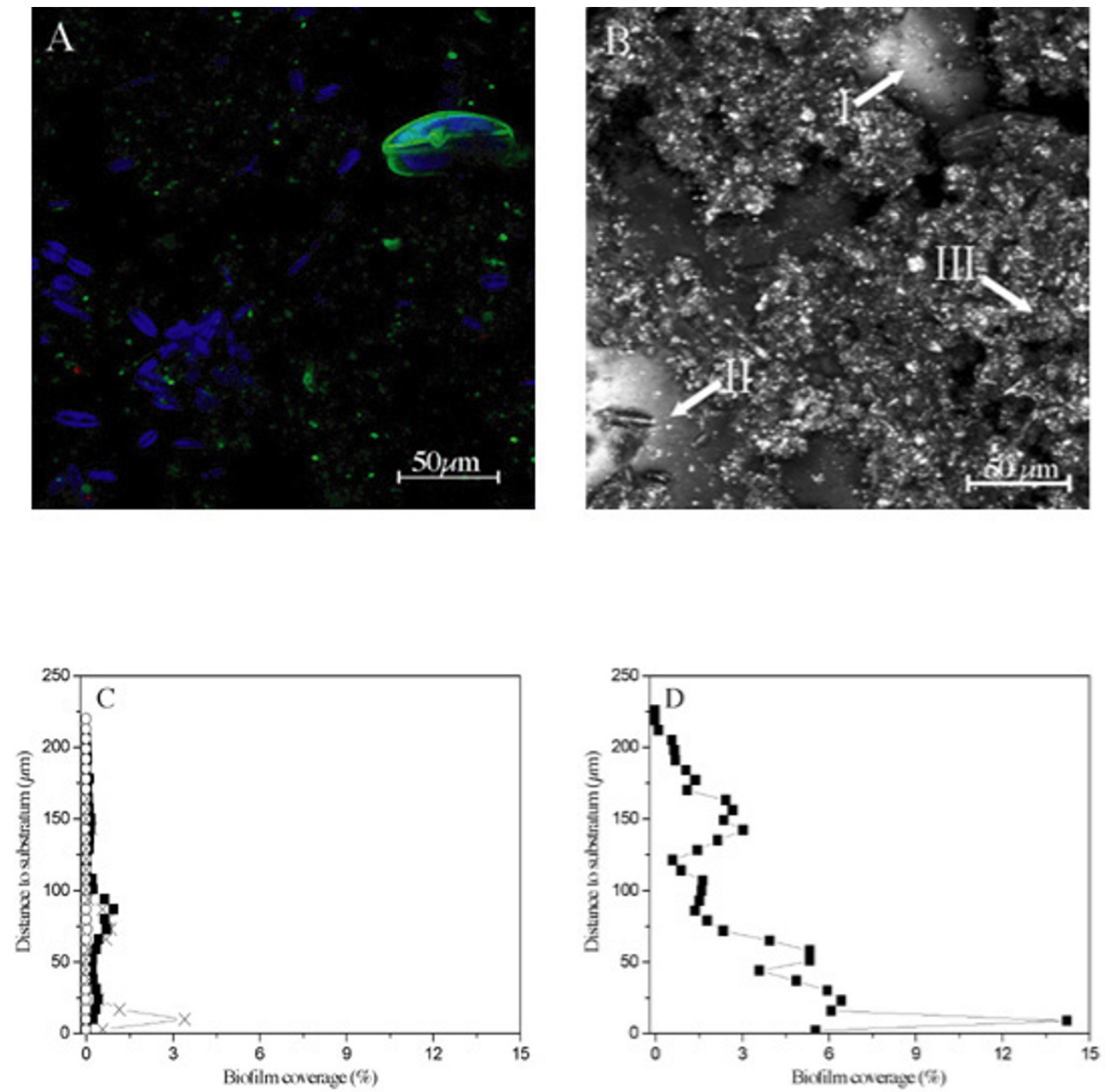

\section{Figure 5}

Maximum projection images of a biofilm grown on glass slides after incubation in natural seawater (Oosterschelde, the Netherlands). Visualization of bacteria, EPS and chlorophyll $a(A)$ and reflection image (B) of a 3 I day old biofilm. The red channel represents bacteria stained with syto64, the green channel represents EPS labeled with ConA and the blue channel shows chlorophyll $a$ autofluorescence. In addition, the depth profiles of biofilm coverage for ConA (filled squares), syto64 (open circles) and chlorophyll $a$ (crosses) are given (C) as well as the depth profile for the reflection signal (D). The CLSM images represent areas of $250 \cdot \mathrm{m} \times 250 \cdot \mathrm{m}$. White arrows indicate the different reflective structures containing bare glass substratum (I), silica frustules of diatoms (II) and amorphous material (III).

species, both qualitatively from visual inspection of images (Fig. 1) and quantitatively from image analysis using PHLIP (Fig. 3A). A possible explanation for this observation is that smaller algae are more opportunistic species adapting more easily, while the bigger ones need a conditioned environment to thrive. During biofilm devel- opment, the different biological components of the biofilms depicted partial stratification (Fig. 1D,E,F). Bacteria were only observed in significant quantities at day 3 and were located close to the substratum. Micro-algae generally inhabited the surface to intermediate layers of the biofilm. The outermost layer of the biofilm almost 
exclusively consisted of EPS, which protruded into the surrounding water (see also Fig. 3B). Micro-stratification has been observed previously in river stream biofilms in which the layer of bacterial was close to the substratum and separated from canopy of micro-algae by an EPSdominated intermediate layer [8]. These results confirm that EPS may be heterogeneously distributed in biofilms. EPS production is considered an important way for biofilm inhabiting organisms to structure their environment [41]. Although EPS was observed as an integral part of the biofilm, this study did not clearly identify the sources of this extracellular material. As mentioned above, twochannel co-localization results from PHLIP analysis (Table 1) show higher values for EPS-bacteria co-localization (ConA-syto64) than for diatom-EPS co-localization (ConA-chla). This slight preferential spatial association of bacteria and EPS suggests that the EPS in this biofilm may be in part of bacterial origin. Visual inspection of images, however, does not provide confirmation that EPS is associated with micro-organisms such as bacteria (Fig. 1A) or diatoms (Fig. 5A). It cannot be excluded that the exopolymeric material that was detected by concanavalin A was not produced by micro-organisms in the biofilm but derived from material attached to particles that were subsequently incorporated into the biofilms (Fig. 5). It is also possible that the organisms had moved deeper into the biofilm leaving the secreted EPS behind. In either case, the stratification of biofilm components and the presence of EPS at the biofilm-water interface are likely to influence mass transfer processes within the biofilm systems [41].

The all-channel analyses showed gradual temporal increases in biovolume and average thickness (Fig. 2). Although this could suggest that development of this biofilm occurred with a rather constant level of heterogeneity, single-channel analyses indicated otherwise. Within the biofilms, bacteria, micro-algae and EPS components followed different dynamics in time (Fig. 3, 4). The appearance of a phototrophic community was preceded by colonization of the glass surface by bacteria which is in agreement with Chan et al [42] who found that bacteria are generally the first colonizers in the development of marine biofilms. During the experiment a clear change was observed from a heterotrophic system dominated by bacteria to an autotrophic system dominated by diatoms. A similar type of dynamics was found for the development of phototrophic freshwater biofilms grown in drinking water basins [9]. Algal and bacterial biomasses were not coupled and bacterial biovolume remained invariably low while micro-algal biomass increased rapidly (Fig. 3A). This is in disagreement with several studies in pelagic systems [43], river stream biofilms $[8,44]$ and marine biofilms [45] where a coupling between algal and bacterial biomass was observed. However, other studies dealing with development of phototrophic biofilms in estuarine systems supported the observation that during phototrophic biofilm development, bacterial biomass remains low $[6,42]$. In marine phytoplankton aggregates, uncoupling of bacteria and algae has been found as a result of grazing on bacteria [46] or insufficient bioavailable algal derived dissolved organic matter [47]. In the present study, the effect of grazing was not specifically investigated. However, microscopic observations showed the presence of organisms in the biofilms that may have utilized biofilm components as a food source. In addition, CLSM analysis of lotic biofilms indicated that grazing greatly impacted on the contribution of biological components and on the morphology of biofilms [11]. Although the structure of the biofilm was generally highly porous, syto64 may not have been penetrated well in the compact parts of the biofilm [9]. Therefore, we could not exclude the possibility that bacterial biovolume was underestimated. This would mean that bacteria were mainly present in EPS aggregates where label penetration is hampered. Other studies suggest that bacteria in natural biofilms are mainly associated with the substratum [48] or distributed in a shell-like manner in outermost regions of biofilms [16]. In addition, previous studies have shown that the contribution of bacteria to phototrophic biofilms was low and typically varied between 0.01 and 5\% $[16,48,49]$. The proportion of bacteria found in this study was in the same range after the phototrophic biofilm was fully developed (1.0-4.9\%). This suggests that the contribution of bacteria in phototrophic biofilms may be low when compared to the algal and EPS components, which represented $20-76 \%$ and $19-77 \%$ of the biofilms, respectively.

The various temporal patterns of individual biofilm components during development of biofilms were also reflected in the morphological characterization.

Roughness coefficients for the bacterial and algal components varied to a large extend whereas those of the EPS matrix were repeatedly high. This supports the view that EPS is an important component defining the structural characteristics of this type of biofilm $[41,50]$. In spite of the different temporal dynamics, a consistent correlation was found between biovolume and roughness of the various biofilm components showing that the biofilm system studied here increased in heterogeneity during its development. However, this does not seem to be a general feature of (phototrophic) biofilms. For example, in phototrophic river stream biofilms roughness coefficients decreased during biofilm development [8]. The mechanisms that determine the morphology of biofilms are currently not understood. It has been observed that morphological development of biofilms is species dependent [24]. Moreover, modeling as well as empirical studies have indicated that biofilm morphology is influ- 


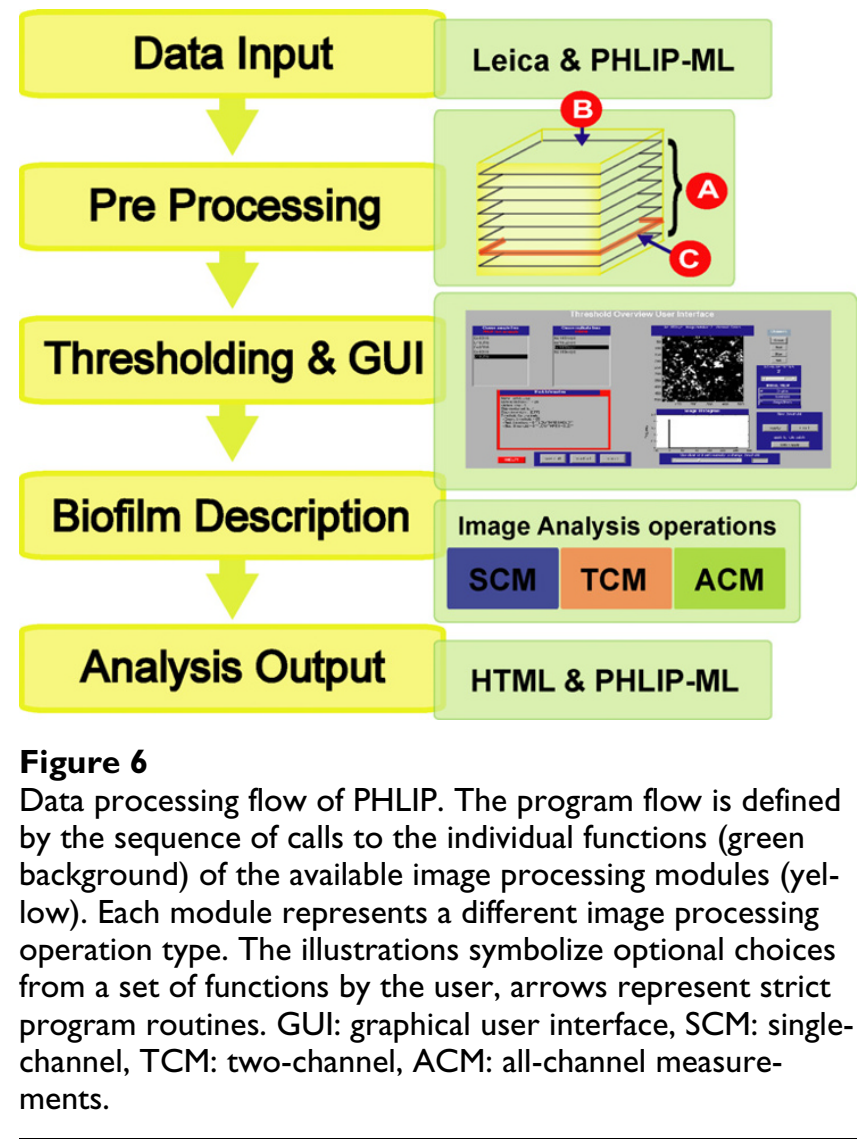

enced by externally imposed constraints including hydrodynamic forces and substrate availability [51-53].

Besides the presence of biological components, the biofilm under study contained considerably amounts of mineral particles as reported for river snow aggregates [19] and river biofilms [8]. It was estimated that as much as $85 \%$ of the biofilm mass was made up by inorganic material, which was either biogenic (e.g. silica, carbonate) or non-biogenic (e.g. silts) in nature. Using chlorophyll $a$ data of the biofilm (not shown) and a Si : chla ratio of 10.9 [54], it was estimated that silica represented approximately $1 \%$ of the mineral mass present in the mature biofilm. This was derived from the phototrophic community that was dominated by diatoms (based on pigment analysis; data not shown). Hence, only a minor part of the inorganic material present in the biofilm was derived from the living phototrophic biomass. Microscope observations in the reflecting mode confirmed this conclusion by showing a predominance of amorphous structures. These structures were visible in the biofilms (Fig. 5B) and may have served to increase the surface area allowing more efficient attachment of micro-organisms to the substratum. In addition, the incorporation of silt particles in biofilm may provide an additional source of nutrients.
This was observed in sedimentary diatom biofilms were a positive feedback was found between diatom growth and silt accumulation [55]. Indeed, increased nutrient concentrations have been reported in estuarine fouling biofilms compared to the ambient water [6].

\section{CLSM quantification with PHLIP}

As shown in previous applications of PHLIP [56,57], we demonstrated here the usefulness of the presented program by the analysis of a large amount of multi-channel CLSM data. In contrast to the recently published program ISA-3D [25], PHLIP is free of charge and the user-friendly program design does not require any training course to understand the program handling. Table 3 in the supplementary material presents a feature overview of the image analysis software mentioned in this article. The recently formed IWA specialist group on biofilm structure aims to evaluate existing image analysis programs (COMSTAT, ISA-3D, PHLIP etc.) and it will be interesting to see the comparison of their performance. We hope that the IWA specialist group will recognize the usefulness of a common CLSM format and help to establish the PHLIP-ML standard within the biofilm community. Although PHLIP constitutes a fully functional image analysis software, the open source project PHLIP intends to further expand the program's capabilities. Current unstable versions offer support for CLSM data from cryo sections including automated stack tilting and incorporate an extension of the fractal dimension parameter into 3D.

To meet the expectations of a novel image analysis software, PHLIP unifies a combination of concepts for automated quantification procedures $[29,30,35]$ and its features address essential issues of morphological quantification for CLSM data of biofilms:

\section{Formalism}

Qualitative morphological analysis of biofilm is often based on subjective concepts. Image processing methods use a quantitative approach to formally and mathematically describe morphological traits [25].

\section{Automation}

PHLIP implements a fully automated image processing procedure, which removes any subjectivity inherited from operator intervention. These batch abilities of PHLIP allow to automatically process large datasets. Although such processing may be computationally demanding, it is executed without the need of user intervention.

\section{Statistical significance of results}

Biofilm development is a stochastic process and, as a consequence, replicate experimental runs never produce the same biofilm structures. This leaves the issue of reproducibility usually open to the morphological quantification 
Table 3: Feature overview of different image analysis software. Comparison of 2 recent published image analysis programs COMSTAT and ISA-3D with PHLIP. Feature information of COMSTAT [24, 26] and ISA-3D [38] was gathered from their reference publications (* data not published, ** data not available).

\begin{tabular}{|c|c|c|c|}
\hline & COMSTAT & ISA-3D & PHLIP \\
\hline Operating systems & Linux, Windows & Windows 2000/XP* & Linux, OSX, Windows \\
\hline Requirements: & Matlab license + toolboxes & Matlab license + toolboxes & Matlab license only \\
\hline Reference & {$[24,26]$} & {$[54]$} & http://www.phlip. org \\
\hline Availability & Obtained free upon request & Must attend workshop $(1500 \$)$ & Free downloadable (open source) \\
\hline Multi-channel capability & No & No & Yes \\
\hline GUI & No & Yes* & Yes \\
\hline CLSM support & $\begin{array}{l}\text { - Leica } \\
\text {-COMSTAT }\end{array}$ & $* *$ & $\begin{array}{l}\text { - Leica } \\
\text { - COMSTAT } \\
\text { - PHLIP-ML } \\
\text { (universal converter) }\end{array}$ \\
\hline Preprocessing & None & - Image inversion & $\begin{array}{l}\text { - Image inversion } \\
\text { - Carrier adjustment } \\
\text { - X- section resolution }\end{array}$ \\
\hline Automatic thresholding & Yes & Yes & Yes (Otsu) \\
\hline Noise reduction: & Connected volume filtration & $* *$ & Connected volume filtration \\
\hline Image analysis parameters: & - Single channel (10) & -Single channel (20) & $\begin{array}{l}\text { - Single channel (6) } \\
\text { - Two channel (2) } \\
\text { - All channel (5) }\end{array}$ \\
\hline Result output & - Text format & - Text format & $\begin{array}{l}\text { - HTML format } \\
\text { - XML format (PHLIP-ML) } \\
\text { - Text-format }\end{array}$ \\
\hline Source code availability & Not available & Not available & Available (open source project) \\
\hline
\end{tabular}

of biofilms from CLSM imaging [21]. The automation capabilities mentioned and the data structures used in the program architecture of PHLIP are specially suited to analyze large datasets with replicates, which is indispensable to obtain statistically significance from an experiment. The analysis of a marine phototrophic biofilm described here used this feature to provide a statistical evaluation of the experimental results.

\section{Standardization}

The presented XML format PHLIP-ML allows the program to work with CLSM data generated by any kind of microscope type. The portability and extensibility of XML offers many advantages to describe the complex nature of biological data and constitutes an important step towards the standardization of methods in biology [58-60]. The PHLIP-ML data structure was designed with the specific intent to serve as a scaffold for further extension to a CLSM standard for sharing data between image analysis programs or image databases. So far, the PHLIP-ML format only describes the microscopic parameters (pixel size, scanning step etc.), provides information about the CLSM data (number of samples/stacks) and stores calculated PHLIP image analysis results. The confocal images themselves only have their filenames identified in the PHLIPML data structure. In the future, a more general and selfcontained approach for a CLSM standard format including a description of experimental procedures should be pursued. Due to the native extensibility of XML, it will be easy to upgrade the PHLIP-ML structure to meet the requirements of such a standard. This will provide a common description of the entire working flow ranging from image analysis to image acquisition process and represent a practical way to transparently reference imaging data to the biofilm community (publications, presentations etc).

\section{Extensibility}

The PHLIP package was built to be easily expandable by additional image processing functions and compatible to new input/output formats. Therefore, the open source project PHILP (made available at [34]) intends to serve as a framework for developers wishing to extend the program and implement their own image processing routines.

\section{Conclusion}

PHLIP analysis was used in this study to reveal the dynamics of three components of a phototrophic biofilm: bacteria, micro-algae and EPS. The observed small contribution of bacteria in mature biofilms compared to algae and EPS suggests a shift from a heterotrophic to an autotrophic system during the biofilm development. Roughness of EPS was one of the morphological parameters measured. Its high value indicated the importance of EPS for the structure of the biofilm.

Enabling the quantification of biofilm morphogenesis as it is described here was the motivation to develop PHLIP. Throughout the analysis process of image segmentation, morphology description and data storage, PHLIP was 
designed to meet the requirements of single- and multichannel capabilities of modern confocal laser scanning microscopy equipment. The PHLIP application software is made public available [53] as an open source implementation in the popular MATLAB scientific environment without the requirement of additional toolboxes. Its modular configuration was specifically developed to facilitate further extension with additional image processing functions in response to particular data analysis requirements and to support other data formats from various confocal microscope models. As examples of the application of image analysis techniques for the quantification of biofilm morphology become more common in the literature, the open structure of PHLIP intends to facilitate the development of novel image analysis procedures.

\section{Availability and requirements}

PHLIP is distributed under an open source license and can be freely downloaded from the PHLIP project webpage [34]. The software comes as a MATLAB package (no toolboxes required) and runs on Linux, OSX and Windows platforms (see also implementation section).

\section{Authors' contributions}

LM and JX programmed the presented image analysis software PHLIP. JB performed the experimental work and quantified the acquired imaging data using PHLIP. LM and JB wrote the manuscript. JA and LS participated in the design and coordination of the project and helped to draft this communication. All authors contributed to the presented study and approved the manuscript.

\section{Acknowledgements}

The authors thank Robin GrayMerod from the Department of Civil and Environmental Engineering at the University of California, Davis, and Barbara Zippel from the Department of River Ecology at UFZ Centre for Environmental Research Leipzig-Halle for their help in testing PHLIP and for useful suggestions. Gwendolyn Pieters is acknowledged for her practical assistance during the biofilm experiments. The experimental work was funded by the Dutch government within the framework of the IOP funded project IZW99|2I. The development of PHLIP was financed by the European Commission through the project PHOBIA (QLK3-CT-2002-0I938). J.B. Xavier thankfully acknowledges financial support from the F.C.T./ M.C.T., Portugal, through the grant SFRH/BPD/I I485/2002. Publication 3745 Netherlands Institute of Ecology (NIOO-KNAW).

\section{References}

I. Donlan RM: Biofilms: Microbial life on surfaces. Emerg Infect Dis 2002, 8:88I-890.

2. Szymanska J: Biofilm and dental unit waterlines. Ann Agric Environ Med 2003, 10:151-157.

3. Chicurel M: Bacterial biofilms and infections. Slimebusters. Nature 2000, 408:284-286.

4. Bryers JD: Processes Contributing to Biofilm Formation: A Review. In First International Conference on Fixed Film Biological Processes: 20-23 April, 1982 Edited by: YC Wu et al. Kings Island, Ohio; 1982:155-183.

5. Mittelman MW: Structure and functional characteristics of bacterial biofilms in fluid processing operations. J Dairy Sci 1998, 8 I:2760-2764.
6. Rao TS, Rani PG, Venugopalan VP, Nair KVK: Biofilm formation in a freshwater environment under photic and aphotic conditions. Biofouling 1997, I I:265-282.

7. Norton TA, Pope J, Veltkamp CJ, Banks B, Howard CV, Hawkins SJ: Using confocal laser scanning microscopy, scanning electron microscopy and phase contrast light microscopy to examine marine biofilms. Aquat Microb Ecol 1998, 16:199-204.

8. Battin TJ, Kaplan LA, Newbold JD, Cheng XH, Hansen C: Effects of current velocity on the nascent architecture of stream microbial biofilms. Appl Environ Microb 2003, 69:5443-5452.

9. Barranguet $C$, van Beusekom SAM, Veuger B, Neu TR, Manders EMM, Sinke J], Admiraal W: Studying undisturbed autotrophic biofilms: still a technical challenge. Aquat Microb Ecol 2004, 34: I-9.

10. Espeland EM, Francoeur SN, Wetzel RG: Influence of algal photosynthesis on biofilm bacterial production and associated glucosidase and xylosidase activities. Microb Ecol 200I, 42:524-530.

II. Lawrence JR, Scharf B, Packroff G, Neu TR: Microscale evaluation of the effects of grazing by invertebrates with contrasting feeding modes on river biofilm architecture and composition. Microb Ecol 2002, 44:199-207.

12. Lawrence JR, Korber DR, Hoyle BD, Costerton JW, Caldwell DE: Optical sectioning of microbial biofilms. J Bacterial I99I, I 73:6558-6567.

13. Lawrence JR, Neu TR: Confocal laser scanning microscopy for analysis of microbial biofilms. Methods Enzymol 1999, 310:131-144.

14. Neu TR, Woelfl S, Lawrence JR: Three-dimensional differentiation of photo-autotrophic biofilm constituents by multichannel laser scanning microscopy (single-photon and twophoton excitation). J Microbiol Meth 2004, 56:161-172.

15. Klausen M, Heydorn A, Ragas P, Lambertsen L, Aaes-Jorgensen A, Molin S, Tolker-Nielsen T: Biofilm formation by Pseudomonas aeruginosa wild type, flagella and type IV pili mutants. Mol Microbiol 2003, 48: I5II-1524.

16. Neu TR, Lawrence JR: Development and structure of microbial biofilms in river water studied by confocal laser scanning microscopy. Microb Ecol 1997, 24: I I-25.

17. Guggenheim B, Guggenheim M, Gmur R, Giertsen E, Thurnheer T: Application of the Zurich biofilm model to problems of cariology. Caries Res 2004, 38:2 I2-222.

18. Moller S, Sternberg C, Andersen JB, Christensen BB, Ramos JL, Givskov M, Molin S: In situ gene expression in mixed-culture biofilms: evidence of metabolic interactions between community members. Appl Environ Microbiol 1998, 64:721-732.

19. Neu TR: In situ cell and glycoconjugate distribution in river snow studied by confocal laser scanning microscopy. Aquat Microb Ecol 2000, $21: 85-95$.

20. Suraruksa B, Nopharatana A, Chaiprasert P, Tanticharoen M, Bhumiratana S: Microbial activity of biofilm during start-up period of anaerobic hybrid reactor at low and high upflow feeding velocity. Water Sci Technol 2003, 48:79-87.

21. Heydorn A, Ersboll BK, Hentzer M, Parsek MR, Givskov M, Molin S: Experimental reproducibility in flow-chamber biofilms. Microbiology 2000, I 46:2409-24I5.

22. Bloem J, Veninga $M$, Shepherd J: Fully automated determination of soil bacterium numbers, cell volumes, and frequencies of dividing cells by confocal laser scanning microscopy and image analysis. Appl Environ Microb 1995, 61:926-936.

23. Liu J, Dazzo FB, Glagoleva O, Yu B, Jain AK: CMEIAS: A Computer-Aided System for the Image Analysis of Bacterial Morphotypes in Microbial Communities. Microb Ecol 200I, 41:173-194.

24. Heydorn A, Nielsen AT, Hentzer M, Sternberg C, Givskov M, Ersboll BK, Molin S: Quantification of biofilm structures by the novel computer program COMSTAT. Microbiology 2000, I 46:2395-2407.

25. Yang X, Beyenal H, Harkin G, Lewandowski Z: Quantifying biofilm structure using image analysis. J Microbiol Meth 2000, 39:109-119.

26. Heydorn A, Ersboll B, Kato J, Hentzer M, Parsek MR, Tolker-Nielsen T, Givskov M, Molin S: Statistical analysis of Pseudomonas aeruginosa biofilm development: impact of mutations in genes involved in twitching motility, cell-to-cell signaling, and stationary-phase sigma factor expression. Appl Environ Microbiol 2002, 68:2008-2017. 
27. Mah TF, Pitts B, Pellock B, Walker GC, Stewart PS, O'Toole GA: A genetic basis for Pseudomonas aeruginosa biofilm antibiotic resistance. Nature 2003, 426:306-310.

28. [http://www.photobiofilms.org]

29. Xavier JB, Schnell A, Wuertz S, Palmer R, White DC, Almeida JS Objective threshold selection procedure (OTS) for segmentation of scanning laser confocal microscope images. J Microbiol Meth 2001, 47:169-180.

30. Xavier JB, White DC, Almeida JS: Automated biofilm morphology quantification from confocal laser scanning microscopy imaging. Water Sci Technol 2003, 47:31-37.

31. [http://phlip.sourceforge.net]

32. Barillot E, Achard F: XML: a lingua franca for science? Trends Biotechnol 2000, I 8:331-333.

33. [http://phlip.sourceforge.net/phlip-ml/]

34. [http://sourceforge.net/projects/phlip/]

35. Kuehn M, Hausner M, Bungartz HJ, Wagner M, Wilderer PA, Wuertz $S$ : Automated confocal laser scanning microscopy and semiautomated image processing for analysis of biofilms. Appl Environ Microbiol 1998, 64:4I I 5-4I 27.

36. Hermanowicz SW, Schindler U, Wilderer P: Fractal structure of biofilms: New tools for investigation of morphology. Water Sci Technol 1995, 32:99-105.

37. Yang X, Beyenal H, Harkin G, Lewandowski Z: Evaluation of biofilm image thresholding methods. Water Res 200I, 35: I|49-1158.

38. Beyenal H, Donovan C, Lewandowski Z, Harkin G: Three-dimensional biofilm structure quantification. J Microbiol Meth 2004 59:395-4I3.

39. Amirand C, Viari A, Ballini JP, Rezaei H, Beaujean N, Jullien D, Kas E, Debey $P$ : Three distinct sub-nuclear populations of HMG-I protein of different properties revealed by co-localization image analysis. J Cell Sci 1998, I I I:355 I-356 I.

40. Sokal RR, Rohlf JR: Biometry: The Principles and Practice of Statistics in Biological Research 3rd edition. W.H. Freeman \& Company; 1997

41. Flemming H-C, Wingender J: Relevance of microbial extracellular polymeric substances (EPSs) - Part II: Technical aspects. Water Sci Technol 200I, 43:9-16.

42. Chan BKK, Chan WKS, Walker G: Patterns of biofilm succession on a sheltered rocky shore in Hong Kong. Biofouling 2003 , I 9:37|-380.

43. Cole J], Findlay S, Pace ML: Bacterial Production in Fresh and Saltwater Ecosystems - a Cross-System Overview. Mar EcolProgs Sers 1988, 43: I- I0

44. Romani AM, Sabater S: Effect of primary producers on the heterotrophic metabolism of a stream biofilm. Freshwater Bio 1999, $41: 729-736$

45. Kerr A, Cowling MJ, Beveridge CM, Smith MJ, Parr ACS, Head RM, Davenport J, Hodgkiess T: The early stages of marine biofouling and its effect on two types of optical sensors. Environ Int 1998 , 24:33I-343

46. Bird DF, Karl DM: Uncoupling of bacteria and phytoplankton during the austral spring bloom in Gerlache Strait, Antarctic Peninsula. Aquat Microb Ecol 1999, 19:13-27.

47. Moran XAG, Estrada M, Gasol JM, Pedros-Alio C: Dissolved primary production and the strength of phytoplankton bacterioplankton coupling in contrasting marine regions. Microb Ecol 2002, 44:217-223

48. Lawrence JR, Neu TR, Swerhone GDW: Application of multiple parameter imaging for the quantification of algal, bacterial and exopolymers components of microbial biofilms. J Microbiol Meth 1998, 32:253-261.

49. Geesey GG, Mutch R, Costerton JW, Green RB: Sessile bacteria: an important component of the microbial population in small mountain streams. Limnol Oceanogr 1978, 23:12 |4- I223.

50. Decho AW: Microbial exopolymer secretions in ocean environments: their role(s) in food webs and marine processes. Oceanogr Mar Biol Rev 1990, 28:73-I53.

51. van Loosdrecht MCM, Eikelboom D, Gjaltema A, Mulder A, Tijhuis L, Heijnen Jl: Biofilm structures. Water Sci Technol I995, 32:35-43.

52. Purevdorj B, Costerton JW, Stoodley P: Influence of hydrodynamics and cell signaling on the structure and behavior of Pseudomonas aeruginosa biofilms. Appl Environ Microb 2002 68:4457-4464
53. van Loosdrecht MC, Heijnen J], Eberl H, Kreft J, Picioreanu C: Mathematical modelling of biofilm structures. Anton Leeuw 2002, 8I:245-256.

54. Sigmon DE, Caboon LB: Comparative effects of benthic microalgae and phytoplankton on dissolved silica fluxes. Aquat Microb Ecol 1997, I 3:275-284.

55. van de Koppel J, Herman PMJ, Thoolen P, Heip CHR: Do alternate stable states occur in natural ecosystems? Evidence from tidal flats. Ecology 200 I, 82:3449-346I

56. GrayMerod R, Hendrickx L, Mueller LN, Xavier JB, Wuertz S: Effect of nucleic acid stain Syto 9 on nascent biofilmarchitecture of Acinetobacter sp. BD4 I3. Water Sci Technol in press.

57. Zippel B, Neu TR: Growth and structure of phototrophic biofilms under controlled light conditions. Water Sci Technol in press.

58. Stanislaus R, Jiang LH, Swartz M, Arthur J, Almeida JS: An XML standard for the dissemination of annotated 2D gel electrophoresis data complemented with mass spectrometry results. BMC Bioinformatics 2004, 5:9.

59. Spellman PT, Miller M, Stewart J, Troup C, Sarkans U, Chervitz S, Bernhart D, Sherlock G, Ball C, Lepage M, Swiatek M, Marks WL, Goncalves J, Markel S, lordan D, Shojatalab M, Pizarro A, White J, Hubley R, Deutsch E, Senger M, Aronow BJ, Robinson A, Bassett D, Stoeckert CJ Jr, Brazma A: Design and implementation of microarray gene expression markup language (MAGE-ML). Genome Biol 2002, 3:1-9.

60. Pedrioli PG, Eng JK, Hubley R, Vogelzang M, Deutsch EW, Raught B, Pratt B, Nilsson E, Angeletti RH, Apweiler R, Cheung K, Costello CE, Hermjakob H, Huang S, Julian RK, Kapp E, McComb ME, Oliver SG, Omenn G, Paton NW, Simpson R, Smith R, Taylor CF, Zhu W, Aebersold $\mathrm{R}$ : A common open representation of mass spectrometry data and its application to proteomics research. Nature Biotechnol 2004, 22:|459-| 466.

Publish with Biomed Central and every scientist can read your work free of charge

"BioMed Central will be the most significant development for disseminating the results of biomedical research in our lifetime. "

Sir Paul Nurse, Cancer Research UK

Your research papers will be:

- available free of charge to the entire biomedical community

- peer reviewed and published immediately upon acceptance

- cited in PubMed and archived on PubMed Central

- yours - you keep the copyright
BioMedcentral 